anales de psicología, 2016, vol. 32, $\mathrm{n}^{\circ} 2$ (mayo), 587-588 http://dx.doi.org/10.6018/analesps.32.2.203841
C Copyright 2016: Servicio de Publicaciones de la Universidad de Murcia. Murcia (España) ISSN edición impresa: 0212-9728. ISSN edición web (http://revistas.um.es/analesps): 1695-2294

\title{
Diferencias entre coeficientes alfa de Cronbach, con muestras y partes pequeñas: Un programa VB
}

\author{
César Merino-Soto
}

Universidad de San Martín de Porres (Lima, Perú).

\begin{abstract}
Resumen: Se presenta un programa informático escrito en VB 6.0, para calcular la diferencia entre coeficientes de consistencia interna (alfa de Cronbach), obtenidos en muestras pequeñas independientes y/o con instrumentos que poseen un número también pequeño de partes o ítems. La comparación de coeficientes de fiabilidad permite identificar posibles diferencias en el tamaño del error de medición en los instrumentos comparados; esta metodología usa el enfoque de prueba de hipótesis, probando la hipótesis nula de igualdad de los coeficientes. Esta condición tiende a ser frecuente en la práctica clínica entre psicólogos y profesiones aliadas, e inclusive en las fases de construcción de instrumentos de medición, por ejemplo en muestras piloto. La técnica aplicada proviene del trabajo de Feldt y Kim (2006), y ofrece una viable e interesante propuesta metodológica que expande el análisis de la fiabilidad de instrumentos de medición psicológicos y educativos. Se presenta las características de su uso, y se discuten las condiciones apropiadas de aplicación.

Palabras clave: fiabilidad; software; metodología; psicometría; alfa de Cronbach.
\end{abstract}

El método más popularizado para estimar un aspecto del error de medición es la consistencia interna (Feldt \& Brennan, 1989), específicamente el coeficiente $\alpha$ (Cronbach, 1951). Sin embargo, para un análisis paramétrico apropiado y preciso con este coeficiente se requieren tamaños muestrales de más de 250 sujetos (Bonnett, 2002, 2003; Charter, 2003). Lo mismo ocurre para examinar las comparaciones entre ellas (Bonnet, 2003; Charter, 2003), que dependen del tamaño de la diferencia para alcanzar un grado de potencia estadística aceptable (Feldt \& Ankenmann, 1998, 1999), y del tamaño muestral para asegurar que la teoría asintótica de la distribución del estadístico sea acertada. Efectivamente, Feldt (1965), y Hakstian y Whalen (1976) derivaron un método basado en la distribución $F$ para comparar dos coeficientes $\alpha$ en muestras independientes, que es eficiente si se cumple una combinación razonable de $(N)(k)=1000$ (Alsawalmeh y Feldt, 1999). Esta combinación de $N$ (tamaño muestra) y k (número de ítems o partes) puede ser problemática en muestras más pequeñas o en un número pequeño de ítems de un instrumento, pues las aproximaciones hacia la distribución $F$ generalmente serán imprecisas.

Para afrontar esto último, Feldt y Kim (2006) propusieron una técnica para comparar coeficientes de fiabilidad en muestras o partes poco numerosas; esto es, cuando el número de partes $(k)$ del instrumento o de participantes en el estudio $(N)$ no superan 2 ó 3 unidades o 100 participantes, respectivamente. La aproximación a la distribución $F$ elaborada por Feldt y Kim se hizo modificando los grados de li-

* Dirección para correspondencia [Correspondence address] César Merino-Soto. Instituto de Investigación de Psicología, (Universidad de San Martín de Porres), Av. Tomás Marsano 242 (5to piso) - Lima 34 (Perú). E-mail: cmerinos@usmp.pe; sikavax@yahoo.com.ar.
Title: Differences between Cronbach's alpha coefficients, with small samples and parts: A VB program.

Abstract: This paper presenting a computer program written in VB 6.0, to calculate the difference between internal consistency coefficients (Cronbach's alpha) obtained in independent small samples and with instruments have a small number of parts or items. The comparison of reliability coefficients allow to identify possible differences in amount of measurement error in instruments; this methodology use the hypothesis testing approach for test the null hypothesis of equally reliability coefficients. This situation is tending to be common in clinical practice between psychologists or allied career, and even in the construction phases of instruments of measurement, for example in pilot samples. The proposed technique is from the work of Feldt and Kim (2006), and offers a viable and interesting methodological proposal that expands the analysis of the reliability of instruments of psychological and educational measurement.

Key words: reliability; software; methodology; psychometry; Cronbach's alpha.

bertad de la distribución $F$ para evaluar su estadístico propuesto.

Para aplicar el procedimiento de Feldt y Kim (2006), en este manuscrito se presenta un programa informático que permite comparar coeficientes de consistencia interna en muestras independientes, en alguna combinación de número de partes de una prueba (ítems) y el tamaño muestral. Los datos requeridos para obtener resultados del programa consisten en los coeficientes $\alpha$ que se compararán, el número de $k$ ítems en cada coeficiente, el tamaño $N$ de la muestra en ambos grupos, y el nivel alfa elegido (convencionalmente, .05 ó .01). Sin embargo, para esta última elección, se puede elegir otro nivel alfa dado que el programa usa un algoritmo complejo de funciones estadísticas. El programa produce resultados usando los coeficientes $a$ sesgados y sin sesgo. Para esto último, se aplica el método de ajuste propuesto por Feldt et al. (1987) para atenuar el sesgo potencial producido por el pequeño tamaño muestral. El software arroja la prueba $W$, el valor $F$ crítico, la probabilidad $p$ y los grados de libertad correspondientes, así como una estimación de la potencia estadística alcanzada; esta última estimación proviene de los procedimientos propuestos por Feldt y Ankenmann (1998, 1999). Hasta la fecha, aparentemente la información de la potencia estadística como un complemento analítico no es rutinariamente considerada en algún análisis de la consistencia interna que se publica, ni en la comparación estadística de coeficientes de fiabilidad con programas informáticos publicados anteriormente (e.g., Lautenschlager, 1989; Lautenschlager \& Meade, 2008; Merino \& Lautenschlager, 2003).

El programa no necesita instalación ni depende de algún archivo dll (Dinamic Link Library) adicional, ya que contiene una librería propia de funciones estadísticas proveniente de Moshier (1989), y traducido al lenguaje VB por Sergey Boch- 
kanov para el proyecto ALGLIB (Bochkanov \& Bystritsky, 2009). Se distribuye como software libre, bajo los términos de la Licencia Pública General GNU, versión 3 o superior

\section{Referencias}

Alsawalmeh, Y. M. y Feldt, L. S. (1999). Testing the equality of independent alpha coefficients adjusted for test length. Educational and Psychological Measurement, 59(3), 373-383. Doi: 10.1177/00131649921969929

Bochkanov, S. y Bystritsky, V. (2009) ALGLIB NET. En línea: (http://www.alglib.net). Acceso: 12 de enero del 2009.

Bonett, D. G. (2002). Sample size requirements for testing and estimating coefficient alpha. Journal of Educational and Behavioral Statistics, 27, 335340. Doi:10.3102/10769986027004335

Bonett, D. G. (2003). Sample size requirements for comparing two alpha coefficients. Applied Psychological Measurement, 27, 72-74. Doi: $10.1177 / 0146621602239477$

Charter, R. A. (2003). Study samples are too small to produce sufficiently precise reliability coefficients. The Journal of General Psychology, 130(2), 117-129. Doi: 10.1080/00221300309601280

Cronbach, L. J. (1951). Coefficient alpha and the internal structure of tests. Psychometrika, 16, 297-334. Doi: 10.1007/BF02310555

Feldt, L. S. y Brennan, R. L. (1989). Reliability. En R. L. Linn (Ed.), Educational measurement (3rd ed., pp. 105-146). New York: Macmillan.

Feldt, L. S. y Kim, S. (2006). Testing the difference between two alpha coefficients with small samples of subjects and raters. Educational and Psychological Measurement, 66(4), 589-600. Doi: 10.1177/0013164405282488

Feldt, L. S. (1965). The approximate sampling distribution of KuderRicharson reliability coefficient twenty. Psychometrika, 30, 357-370. Doi: 10.1007/BF02289499 (ver licencia en http://gnu.org/licenses/). Se puede solicitar una copia gratuita del programa a las direcciones de contacto del autor.

Feldt, L. S. y Ankenmann, R. D. (1998). Appropriate sample size for comparing alpha reliabilities. Applied Psychological Measurement, 22, 170-178. Doi: $1177 / 01466216980222006$

Feldt, L. S. y Ankenmann, R. D. (1999). Determining sample size for a test of the equality of alpha coefficients when the number of part-tests is small. Psychological Methods, 4, 366-377. Doi: 10.1007/BF02294264

Hakstian, A. R. y Whalen, T. E. (1976). A k-sample significance test for independent Alpha coefficients. Psychometrika, 41, 219-231. Doi: 10.1007/BF02291840

Lautenschlager, G. J. y Meade, A. W. (2008). AlphaTest: A windows program for tests of hypotheses about coefficient alpha. Applied Psychological Measurement, 23, 502-503. Doi: 10.1177/0146621607312307

Lautenschlager, G. J. (1989). Alphatest, testing for differences in values of coefficient alpha. Applied Psychological Measurement, 13 (3) 284. Doi: $10.1177 / 0146621607312307$.

Merino, C. \& Lautenschlager, G. J. (2003). Comparación estadística de la confiabilidad alfa de Cronbach: aplicaciones en la medición educacional y psicológica. Revista de Psicología de la Universidad de Chile, 12(2), 129-139.

Moshier, S.L. (1989). Methods and programs for mathematical functions. Englewood Cliffs, NJ: Prentice-Hall.

(Articulo recibido: 31-07-2014; revisado: 26-09-2014; aceptado: 08-02-2015) 